\title{
Eficiência de métodos para o cálculo de coeficientes do tanque classe A na estimativa da evapotranspiração de referência ${ }^{1}$
}

\author{
Paulo César Ribeiro da Cunha ${ }^{2}$, Jorge Luiz do Nascimento ${ }^{3}$, Pedro Marques da Silveira ${ }^{4}$, José Alves Júnior ${ }^{3}$
}

\begin{abstract}
Efficiency of methods for calculating class A pan coefficients to estimate evapotranspiration reference

The class A pan coefficients (Kp) can be determined with several methods, most based on wind speed, relative humidity and conditions and extension of the border which circles the pan. This study aimed at evaluating the performance of methods used for estimating the $\mathrm{Kp}$, as well as an average and constant $\mathrm{Kp}(0.70)$, to evaluate the reference evapotranspiration $\left(\mathrm{ET}_{0}\right)$ under Brazilian Savannah conditions, in Santo Antônio de Goiás, Goiás State, Brazil. The methods were assessed on an annual basis and during the dry and rainy seasons. The $\mathrm{ET}_{0}$ resulting from the class A pan evaporation, multiplied by the Kp values estimated with the aid of the Doorenbos \& Pruitt (1977), Cuenca (1989), Snyder (1992), Pereira et al. (1995) and Allen et al. (1998) methods, were compared with the $\mathrm{ET}_{0}$ estimated by using the Penman-Montheit equation. The coefficients of determination $\left(\mathrm{r}^{2}\right)$, correlation $(\mathrm{r})$, Willmott index of agreement (d) and performance (c), as well as the mean and maximum absolute errors, were also estimated. Considering the weather conditions during the experiment, the best Kp method to estimate the annual $\mathrm{ET}_{0}$ was the one proposed by Pereira et al. (1995). For the dry season, the method proposed by Cuenca (1989) performed better, while, for the rainy season, all methods showed a low performance, with the Pereira et al. (1995) method being the most efficient one. The use of a constant Kp of 0.70 resulted in a good performance and showed to be a practical option, however, it is necessary to determine it where it will be applied.
\end{abstract}

KEY-WORDS: Penman-Monteith equation; climate; irrigation management.

\section{INTRODUÇÃO}

O processo de ocorrência simultânea da evaporação da água do solo e da transpiração da água pela planta é denominado evapotranspiração, sendo uma

\section{RESUMO}

Os valores do coeficiente do tanque classe A $(\mathrm{Kp})$ podem ser determinados por vários métodos. A maioria baseiase na velocidade do vento, umidade relativa e condições e extensão da bordadura que circunda o tanque. Este estudo objetivou avaliar o desempenho de métodos de estimativa do Kp e o uso de um Kp médio e constante $(0,70)$, na avaliação da evapotranspiração de referência $\left(\mathrm{ET}_{0}\right)$, sob condições de Cerrado, em Santo Antônio de Goiás (GO). Os métodos foram avaliados nos períodos anual, seco e chuvoso. As ET Ebtidas $_{0}$ por meio da evaporação do tanque, multiplicadas pelos valores de Kp estimados com os métodos de Doorenbos \& Pruitt (1977), Cuenca (1989), Snyder (1992), Pereira et al. (1995) e Allen et al. (1998), foram comparadas com a $\mathrm{ET}_{0}$ estimada pelo modelo de Penman-Montheit. Foram determinados os coeficientes de determinação $\left(\mathrm{r}^{2}\right)$, correlação $(\mathrm{r})$, concordância (d) de Willmott e de desempenho (c) e, também, os erros médios e máximos absolutos. Para as condições climáticas nas quais se realizou o trabalho, o melhor método de determinação do Kp, para estimativa da $\mathrm{ET}_{0}$ no período anual, foi o de Pereira et al. (1995). Para o período seco, destacou-se o método proposto por Cuenca (1989) e, para o período chuvoso, todos os métodos apresentaram baixos desempenhos, sendo o de Pereira et al. (1995) o mais eficiente. A adoção de um Kp fixo e constante de 0,70 resultou em bom desempenho, sendo uma opção bastante prática, porém, faz-se necessária a sua determinação no local onde será aplicado.

PALAVRAS-CHAVE: Equação de Penman-Monteith; clima; manejo da irrigação.

parcela da água cedida à atmosfera. A evapotranspiração é o principal componente de saída do balanço hídrico, sendo um processo de grande relevância para o manejo agrícola. Para Pereira et al. (1997), a evapotranspiração é controlada, principalmente, pela

1. Trabalho recebido em jun./2012 e aceito para publicação em abr./2013 (nº registro: PAT 18844).

2. Instituto Federal de Educação, Ciência e Tecnologia Goiano (IFG), Campus Urutaí, Urutaí, GO, Brasil. E-mail: pcdacunha@hotmail.com.

3. Universidade Federal de Goiás (UFG), Escola de Agronomia, Goiânia, GO, Brasil. E-mails: jln.agro@gmail.com, josealvesufg@yahoo.com.br.

4. Empresa Brasileira de Pesquisa Agropecuária, Embrapa Arroz e Feijão, Santo Antônio de Goiás, GO, Brasil.

E-mail: pmarques@cnpaf.embrapa.br. 
disponibilidade de energia, pela demanda atmosférica e pelo suprimento de água do solo às plantas.

Na escolha de um método para a determinação da evapotranspiração, devem ser levadas em consideração a praticidade e precisão, pois, apesar de os métodos teóricos e micrometeorológicos serem baseados em princípios físicos, apresentam limitações, principalmente quanto à instrumentação, o que pode restringir a sua utilização.

Apesar de algumas desvantagens, como a superexposição às condições ambientais, a facilidade de acesso de animais e ao fato de a evaporação ocorrer, também, no período noturno (Sentelhas 2001), o tanque classe A é tido como um dos métodos indiretos passíveis de utilização no manejo de áreas irrigadas, em virtude do custo relativamente baixo. No entanto, sua adoção no manejo exige que sejam observados alguns aspectos, principalmente quanto à escolha do coeficiente do tanque classe A (Kp) a ser utilizado. Sentelhas \& Folegatti (2003) ainda alertam para a necessidade de ajuste do Kp, para as condições locais de aplicação do método.

Para estimar a evapotranspiração de referência $\left(\mathrm{ET}_{0}\right)$, com base na evaporação do tanque classe A (ECA), multiplica-se o valor da ECA por um coeficiente do tanque (Kp), determinando-se, assim, a ET .

Existem vários métodos para se estimar o valor do Kp, sendo, praticamente, todos eles baseados em dados diários médios de velocidade do vento $(\mathrm{u})$, umidade relativa $(\mathrm{H})$ e no tipo e tamanho da bordadura que circunda o tanque classe A (F) (Doorenbos \& Pruitt 1977, Mendonça et al. 2006). Contudo, é necessário que se estabeleça qual metodologia é capaz de propiciar a melhor estimativa da $\mathrm{ET}_{0}$ com base na ECA, para a localidade desejada (Conceição 2002).

Doorenbos \& Pruitt (1977) apresentam uma tabela com valores de $\mathrm{Kp}$ variando de 0,40 a 0,85 , dependentes das condições climáticas e da bordadura que circunda o tanque. Cuenca (1989) modificou a equação proposta por Frevert et al. (1983) e sugeriu uma equação polinomial para estimar valores diários de Kp. Snyder (1992) considerou a equação proposta por Cuenca (1989) muito complexa e com resultados insatisfatórios, para algumas condições climáticas, quando comparada aos coeficientes tabelados propostos por Doorenbos \& Pruitt (1977), então, propôs um método de regressão linear múltipla para estimar o coeficiente do tanque. O método proposto por Pereira et al. (1995) baseia-se na temperatura do ar e na constante psicrométrica. Allen et al. (1998) apresen- taram uma equação de regressão derivada da tabela de Doorenbos \& Pruitt (1977), para se estimar o Kp com base na velocidade do vento, umidade relativa e no tipo e extensão da bordadura em volta do tanque.

Mesmo com vários métodos para se calcular o Kp, Sentelhas \& Folegatti (2003) afirmam que são poucos os trabalhos que avaliaram a precisão e desempenho destes métodos sob as condições climáticas brasileiras, principalmente sob condições de Cerrado, onde se concentram extensas áreas irrigadas por pivôs centrais.

Diante da importância de se estimar corretamente a $\mathrm{ET}_{0}$, o presente estudo objetivou avaliar o desempenho de métodos de estimativa do $\mathrm{Kp}$, dos valores tabelados propostos por Doorenbos \& Pruitt (1977) e o uso de um valor fixo e constante $(0,70)$, na estimativa da $\mathrm{ET}_{0}$ diária, a partir da evaporação do tanque classe A, sob as condições climáticas da sede da Embrapa Arroz e Feijão, no município de Santo Antônio de Goiás (GO).

\section{MATERIAL E MÉTODOS}

O trabalho foi desenvolvido com informações meteorológicas obtidas na estação automática da Embrapa Arroz e Feijão, instalada em Santo Antônio de Goiás (GO) (16 $28^{\circ} \mathrm{S}, 49^{\circ} 17^{\prime} \mathrm{W}$ e $823 \mathrm{~m}$ de altitude). Em função da disponibilidade de dados, optou-se pela utilização de dados diários do ano de 2007. Trabalhou-se, em média, com doze valores diários, em cada mês. Conforme Mendonça et al. (2006), foram excluídos os dados referentes aos dias em que houve ocorrência de altas precipitações (superiores a 30,0 mm diários), assim como ao dia posterior, em que foram observados dados discrepantes, incompletos e até inconsistentes, visando à obtenção de agrupamentos de dados mais representativos.

$\mathrm{A} \mathrm{ET}_{0}$ diária considerada como padrão foi estimada utilizando-se o modelo de Penman-Monteith (Allen et al. 1998), com os dados climatológicos obtidos na estação automática. Calculou-se a ET ${ }_{0}$ pelo método do tanque classe $\mathrm{A}$, instalado sobre gramado com 10,0 m de bordadura, com os coeficientes do tanque obtidos pelos métodos propostos por Doorenbos \& Pruitt (1977), Snyder (1992), Cuenca (1989), Pereira et al. (1995) e Allen et al. (1998) e Kp fixo em 0,7 , valor correspondente à média anual obtida com o método de Doorenbos \& Pruitt (1977). Os resultados foram comparados com a $\mathrm{ET}_{0}$ estimada pelo modelo de Penman-Monteith. 
O método proposto por Doorenbos \& Pruitt (1977) é o mais comumente apresentado na literatura. O valor do Kp é dado em função da velocidade do vento, umidade relativa média e das condições e tamanho da bordadura que circunda o tanque (Tabela 1).

O método proposto por Snyder (1992) é baseado em uma equação de regressão linear múltipla: $\mathrm{Kp}=0,482+0,024 \mathrm{Ln}(\mathrm{F})-0,000376 \mathrm{u}+0,0045 \mathrm{H}$, em que $\operatorname{Ln}(\mathrm{F})$ é o logaritmo natural de $F$, que é a bordadura da área que circunda o tanque, considerada igual a $10,0 \mathrm{~m}$ de grama, no presente trabalho; " $u$ " a velocidade do vento $\left(\mathrm{km} \mathrm{dia}^{-1}\right)$, medida a 2,0 $\mathrm{m}$ do solo; e $\mathrm{H}$ a umidade relativa média, em percentagem.

O método de Cuenca (1989) baseia-se em uma equação polinomial para estimar valores diários de Kp, com base na velocidade do vento, umidade relativa e no tipo e extensão da bordadura em volta do tanque: $\mathrm{Kp}=0,475-2,4 \times 10^{-4} \mathrm{u}+5,16 \times 10^{-3} \mathrm{H}+$ $1,18 \times 10^{-3} \mathrm{~F}-1,6 \times 10^{-5} \mathrm{H}^{2}-1,01 \times 10^{-6} \mathrm{~F}^{2}-8,0 \times$ $10^{-9} \mathrm{H}^{2} \mathrm{u}-1,0 \times 10^{-8} \mathrm{H}^{2} \mathrm{~F}$, em que " $\mathrm{u}$ " é a velocidade do vento $\left(\mathrm{m} \mathrm{s}^{-1}\right)$, medida a $2,0 \mathrm{~m}$ do solo; $\mathrm{F}$ é dado pela distância da bordadura que circunda o tanque, considerada igual a $10,0 \mathrm{~m}$ de grama, no presente trabalho; e $\mathrm{H}$ a umidade relativa média.

O modelo de Pereira et al. (1995) baseia-se na temperatura do ar e na constante psicrométrica:

$$
\operatorname{Kp}=\operatorname{Kpmáx} \frac{(s+\gamma)}{\left[s+\gamma\left(1+\frac{r_{c}}{r_{a}}\right)\right]}
$$

em que considera-se Kpmáx de 0,85 , como observado por Pereira et al. (1997), sendo este o maior valor encontrado na tabela proposta por Doorenbos \& Pruitt (1977); "s" é a tangente à curva de pressão de saturação na temperatura do ar $\left(\mathrm{kPa}^{\circ} \mathrm{C}^{-1}\right) ; \gamma$ a constante psicrométrica $\left(\mathrm{kPa}^{\circ} \mathrm{C}^{-1}\right)$; e $\mathrm{r}_{\mathrm{c}} / \mathrm{r}_{\mathrm{a}}$ a relação entre a resistência do dossel foliar da grama à difusão do vapor d'água $\left(r_{c}\right)$ e a resistência aerodinâmica para a troca do vapor d'água de uma superfície evaporante $\left(\mathrm{r}_{\mathrm{a}}\right)$. Para se obter tal relação, procedeu-se conforme sugerido por Allen et al. (1998), que estabelecem uma relação empírica com a velocidade do vento (u), em $\mathrm{m} \mathrm{s}^{-1}$, medida a 2,0 m do solo, conforme a seguinte equação:

$$
\frac{\mathrm{r}_{\mathrm{c}}}{\mathrm{r}_{\mathrm{a}}}=0,34 \mathrm{u}
$$

O método recomendado pela $\mathrm{FAO} / 56$ (Allen et al. 1998) é apresentado na equação a seguir, que consiste em uma equação de regressão derivada da tabela de Doorenbos \& Pruitt (1977): Kp = $0,108-0,0286 u+0,0422 \operatorname{Ln}(\mathrm{F})+0,1434 \mathrm{Ln}(\mathrm{H})-$ $0,000631[\operatorname{Ln}(F)]^{2} \operatorname{Ln}(\mathrm{H})$, em que " $u$ " é a velocidade

Tabela 1. Valores de coeficiente do tanque propostos por Doorenbos \& Pruitt (1977), em função da velocidade do vento, umidade

\begin{tabular}{|c|c|c|c|c|c|c|c|}
\hline \multirow{2}{*}{$\begin{array}{l}\text { Velocidade do } \\
\text { vento } \\
\left(\mathrm{m} \mathrm{s}^{-1}\right)\end{array}$} & \multirow{2}{*}{$\begin{array}{c}\text { Posição } \\
\text { do tanque } \mathrm{R}^{*} \\
\text { (m) }\end{array}$} & \multicolumn{3}{|c|}{$\begin{array}{c}\text { Tanque circundado por grama } \\
\text { Umidade relativa média }\end{array}$} & \multicolumn{3}{|c|}{$\begin{array}{l}\text { Tanque circundado por solo nu } \\
\text { Umidade relativa média }\end{array}$} \\
\hline & & $\begin{array}{l}\text { Baixa } \\
<40 \%\end{array}$ & $\begin{array}{c}\text { Média } \\
40-70 \%\end{array}$ & $\begin{array}{c}\text { Alta } \\
>70 \%\end{array}$ & $\begin{array}{l}\text { Baixa } \\
<40 \%\end{array}$ & $\begin{array}{c}\text { Média } \\
40-70 \%\end{array}$ & $\begin{array}{c}\text { Alta } \\
>70 \%\end{array}$ \\
\hline \multirow{4}{*}{$\begin{array}{l}\text { Fraco } \\
<2\end{array}$} & 0 & 0,55 & 0,65 & 0,75 & 0,70 & 0,80 & 0,85 \\
\hline & 10 & 0,65 & 0,75 & 0,85 & 0,60 & 0,70 & 0,80 \\
\hline & 100 & 0,70 & 0,80 & 0,85 & 0,55 & 0,65 & 0,75 \\
\hline & 1000 & 0,75 & 0,85 & 0,85 & 0,50 & 0,60 & 0,70 \\
\hline \multirow{4}{*}{$\begin{array}{l}\text { Moderado } \\
\quad 2-5\end{array}$} & 0 & 0,50 & 0,60 & 0,65 & 0,65 & 0,75 & 0,80 \\
\hline & 10 & 0,60 & 0,70 & 0,75 & 0,55 & 0,65 & 0,70 \\
\hline & 100 & 0,65 & 0,75 & 0,80 & 0,50 & 0,60 & 0,65 \\
\hline & 1000 & 0,70 & 0,80 & 0,80 & 0,45 & 0,55 & 0,60 \\
\hline \multirow{4}{*}{$\begin{array}{c}\text { Forte } \\
5-8\end{array}$} & 0 & 0,45 & 0,50 & 0,60 & 0,60 & 0,65 & 0,70 \\
\hline & 10 & 0,55 & 0,60 & 0,65 & 0,50 & 0,55 & 0,65 \\
\hline & 100 & 0,60 & 0,65 & 0,75 & 0,45 & 0,50 & 0,60 \\
\hline & 1000 & 0,65 & 0,70 & 0,75 & 0,40 & 0,45 & 0,55 \\
\hline \multirow{4}{*}{$\begin{array}{l}\text { Muito forte } \\
\quad>8\end{array}$} & 0 & 0,40 & 0,45 & 0,50 & 0,50 & 0,60 & 0,65 \\
\hline & 10 & 0,45 & 0,55 & 0,60 & 0,45 & 0,50 & 0,55 \\
\hline & 100 & 0,50 & 0,60 & 0,65 & 0,40 & 0,45 & 0,50 \\
\hline & 1000 & 0,55 & 0,60 & 0,65 & 0,35 & 0,40 & 0,45 \\
\hline
\end{tabular}
relativa média do ar e do meio circundante ao tanque.

Fonte: Doorenbos \& Pruitt (1977). * Menor distância do centro do tanque ao limite da bordadura. 
do vento a 2,0 $\mathrm{m}$ do solo, em $\mathrm{m} \mathrm{s}^{-1}$; $\mathrm{F}$ é dado pela distância da bordadura que circunda o tanque, sendo de 10,0 m de grama, no presente trabalho; e $\mathrm{H}$ a umidade relativa média.

Com base na média anual dos valores tabelados propostos por Doorenbos \& Pruitt (1977), para o ano de 2007, optou-se pela avaliação de um valor fixo e constante $(0,7)$. Os métodos de estimativa, assim como o uso de um valor fixo e constante do $\mathrm{Kp}$, foram avaliados quanto ao desempenho para estimativa da $\mathrm{ET}_{0}$ a partir da ECA, considerando-se o período anual, período chuvoso (outubro a março) e período de seca (abril a setembro).

A avaliação do desempenho dos métodos de cálculo do Kp na estimativa da $\mathrm{ET}_{0}$, a partir da evaporação do tanque classe $\mathrm{A}$, foi feita empregando-se análise de correlação, índice de concordância de Willmott (d) (Willmott et al. 1985), erro médio absoluto (EMA), erro máximo absoluto (EMAX) e eficiência do método (EF) (Zacharias et al. 1996), conforme as seguintes equações:

$$
\begin{aligned}
& \mathrm{d}=1-\frac{\sum_{\mathrm{i}=1}^{\mathrm{n}}\left(\mathrm{O}_{\mathrm{i}}-\mathrm{E}_{\mathrm{i}}\right)^{2}}{\sum_{\mathrm{i}=1}^{\mathrm{n}}\left(\left|\mathrm{E}_{\mathrm{i}}-\overline{\mathrm{O}}\right|+\left|\mathrm{O}_{\mathrm{i}}-\overline{\mathrm{O}}\right|\right)^{2}} \\
& \mathrm{EMA}=\frac{1}{\mathrm{n}} \sum_{\mathrm{i}=1}^{\mathrm{n}}\left|\mathrm{O}_{\mathrm{i}}-\mathrm{E}_{\mathrm{i}}\right| \\
& \mathrm{EMAX}=\operatorname{MAX}\left(\left|\mathrm{O}_{\mathrm{i}}-\mathrm{E}_{\mathrm{i}}\right|\right)_{\mathrm{i}=1}^{\mathrm{n}} \\
& \mathrm{EF}=\frac{\sum_{\mathrm{i}=1}^{\mathrm{n}}\left(\mathrm{O}_{\mathrm{i}}-\overline{\mathrm{O}}\right)^{2}-\sum_{\mathrm{i}=1}^{\mathrm{n}}\left(\mathrm{O}_{\mathrm{i}}-\mathrm{E}_{\mathrm{i}}\right)^{2}}{\sum_{\mathrm{i}=1}^{\mathrm{n}}\left(\mathrm{O}_{\mathrm{i}}-\overline{\mathrm{O}}\right)^{2}}
\end{aligned}
$$

em que $\mathrm{O}_{\mathrm{i}}$ é o valor obtido por meio da ECA, $\mathrm{E}_{\mathrm{i}} \mathrm{o}$ valor estimado pelo modelo padrão e $\overline{\mathrm{O}}$ a média dos valores observados.

Efetuou-se a comparação dos métodos e da adoção de Kp fixo segundo estudo de análise de correlação entre a $\mathrm{ET}_{0}$ estimada pelo método de Penman-Monteith e a $\mathrm{ET}_{0}$ estimada por meio da ECA, multiplicada pelo Kp fixo, e entre a $\mathrm{ET}_{0}$ padrão obtida por meio da ECA e os valores de Kp estimados pelos métodos avaliados neste trabalho. Analisaram-se os coeficientes angular (b), de correlação (r) e de determinação $\left(\mathrm{r}^{2}\right)$ obtidos em cada caso. Os valores dos coeficientes de correlação encontrados foram classificados seguindo-se a classificação proposta por Hopkins (2000) (Tabela 2).

Com o intuito de dar suporte à análise, determinou-se o índice de desempenho (c) (Camargo \& Sentelhas 1997), que é o resultado do produto entre o coeficiente de correlação de Pearson (r) e o índice de exatidão de Willmott (d), cujos critérios de interpretação encontram-se especificados na Tabela 3.

\section{RESULTADOS E DISCUSSÃO}

Ao analisar os valores dos coeficientes de determinação $\left(\mathrm{r}^{2}\right)$ (Tabela 4), que indicam as precisões das estimativas, observou-se que o Kp tabelado proposto por Doorenbos \& Pruitt (1977) e Snyder (1992) apresentou baixo desempenho, em relação aos demais métodos. O mesmo foi constatado por Sentelhas \& Folegatti (2003), que avaliaram o desempenho de seis métodos de estimativa do Kp, para o período anual, com base na $\mathrm{ET}_{0}$ diária obtida pelo tanque classe A e a medida em um lisímetro de pesagem, durante 112 dias, entre dezembro de 1995 e dezembro de 1996, em Piracicaba (SP). No entanto, eles obtiveram valores de coeficientes de determinação superiores aos encontrados neste trabalho, o que, em parte, pode-se atribuir ao fato de terem utilizado menor conjunto de dados, o que possibilita a eliminação de valores dispersivos.

Tabela 2. Classificação dos valores do coeficiente de correlação de Pearson (r).

\begin{tabular}{cc}
\hline Coeficiente de correlação $(\mathrm{r})$ & Classificação \\
\hline 0,0 a 0,1 & Muito baixa \\
0,1 a 0,3 & Baixa \\
0,3 a 0,5 & Moderada \\
0,5 a 0,7 & Alta \\
0,7 a 0,9 & Muito alta \\
0,9 a 1,0 & Quase perfeita \\
\hline
\end{tabular}

Fonte: Hopkins (2000).

Tabela 3. Critérios de interpretação do índice de desempenho "c".

\begin{tabular}{cc}
\hline Índice de desempenho "c" & Classificação \\
\hline$>0,85$ & Ótimo \\
$0,76-0,85$ & Muito bom \\
$0,66-0,75$ & Bom \\
$0,61-0,65$ & Mediano \\
$0,51-0,60$ & Sofrível \\
$0,41-0,50$ & Mal \\
$\leq 0,40$ & Péssimo \\
\hline
\end{tabular}

Fonte: Camargo \& Sentelhas (1997). 
Tabela 4. Análise estatística para avaliação dos métodos de estimativa e cálculo do coeficiente do tanque (Kp), para estimativa da evapotranspiração de referência diária $\left(\mathrm{ET}_{0}\right)$ pelo método do tanque classe $\mathrm{A}$, no período anual (Santo Antônio de Goiás, $\mathrm{GO}, 2007)$.

\begin{tabular}{|c|c|c|c|c|c|c|}
\hline \multirow{2}{*}{ Método } & \multirow{2}{*}{$\mathrm{b}$} & \multirow{2}{*}{$r^{2}$} & \multirow{2}{*}{$\mathrm{d}$} & EMA & EMAX & \multirow{2}{*}{$\mathrm{EF}$} \\
\hline & & & & 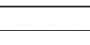 & & \\
\hline Doorenbos \& Pruitt (1977) & 0,88 & 0,48 & 0,83 & 0,70 & 3,01 & 0,43 \\
\hline Snyder (1992) & 0,88 & 0,46 & 0,82 & 0,72 & 3,07 & 0,34 \\
\hline Cuenca (1989) & 0,90 & 0,55 & 0,87 & 0,64 & 2,57 & 0,55 \\
\hline Pereira et al. (1995) & 0,86 & 0,54 & 0,86 & 0,71 & 3,31 & 0,61 \\
\hline Allen et al. (1998) & 0,91 & 0,55 & 0,88 & 0,63 & 2,48 & 0,57 \\
\hline $\operatorname{Kp}$ fixo $(0,7)$ & 0,92 & 0,56 & 0,90 & 0,60 & 2,61 & 0,71 \\
\hline
\end{tabular}

b: coeficiente angular; $\mathrm{r}^{2}$ : coeficiente de determinação; d: índice de exatidão de Willmott; EMA: erro médio absoluto; EMAX: erro máximo absoluto; EF: eficiência do método.

Esses resultados diferem dos observados por Conceição (2002), que avaliou três dos métodos apresentados neste trabalho, em escala mensal, para as condições do noroeste do Estado de São Paulo, com o método proposto por Snyder (1992) apresentando melhor desempenho para estimativa da $\mathrm{ET}_{0}$ a partir da ECA, naquelas condições. Gundekar et al. (2008), em condições de clima semiárido, na Índia, obtiveram resultados totalmente diferentes dos observados neste trabalho, tendo verificado que o método de Snyder (1992) apresentou o melhor desempenho na estimativa do $\mathrm{Kp}$. Tais resultados demonstram a necessidade de avaliação dos métodos disponíveis para o cálculo do Kp para a localidade específica onde se pretende aplicá-los.

$\mathrm{O}$ valor médio anual do Kp calculado para o período anual, a partir da relação entre a $\mathrm{ET}_{0}$ diária estimada pelo método padrão Penman-Monteith e a ECA, foi de 0,7021 . No período seco (abril a setembro), o Kp médio foi de 0,6811 e o maior valor de $\mathrm{Kp}(0,7229)$ foi obtido durante o período chuvoso (outubro a março). Conceição (2002) também obteve coeficientes médios do tanque maiores para o período chuvoso $(0,80)$ e menores no período seco $(0,70)$, com Kp médio anual de 0,74.

Para a estimativa da $\mathrm{ET}_{0}$ diária, durante o período anual, os métodos que apresentaram os maiores coeficientes de determinação foram os de Cuenca (1989), Allen et al. (1998) e Pereira et al. (1995). Mendonça et al. (2006) também observaram bom desempenho dos métodos de Cuenca (1989) e Allen et al. (1998), em trabalho semelhante, realizado em con-

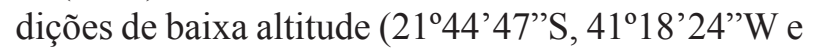
$11 \mathrm{~m}$ de altitude), em Campos dos Goytacazes (RJ). O método de Cuenca (1989) também se destacou como o mais eficiente no trabalho de Sentelhas \& Folegatti (2003), em Piracicaba (SP), tendo estes verificado alta eficiência do método proposto por Pereira et al. (1995).

Observou-se que todos os métodos apresentaram coeficiente angular (b) inferior a um, o que evidencia que o método do tanque classe $\mathrm{A}$, independentemente do Kp utilizado, superou os valores de $\mathrm{ET}_{0}$ estimados pelo método padrão FAO (Penman-Monteith). O mesmo foi observado por Conceição (2002), Sentelhas \& Folegatti (2003) e Mendonça et al. (2006).

$\mathrm{O}$ erro médio absoluto das estimativas de $\mathrm{ET}_{0}$ obtidas pelo método do tanque classe $\mathrm{A}$, com os $\mathrm{Kp}$ avaliados em relação às obtidas com o modelo padrão, variou de $0,63 \mathrm{~mm}$ dia $^{-1}$ a $0,72 \mathrm{~mm} \mathrm{dia}^{-1}$, segundo os métodos de Allen et al. (1998) e Snyder (1992), respectivamente (Tabela 4). Estes resultados se assemelham aos obtidos por Sentelhas \& Folegatti (2003), que observaram maior erro médio absoluto $(0,64)$ com o modelo de Snyder (1992). Resultado semelhante foi obtido por Mendonça et al. (2006).

O método que apresentou melhor desempenho para determinação do Kp, visando a converter a ECA em $\mathrm{ET}_{0}$, foi o proposto por Pereira et al. (1995) $(d=0,86$ e EF =0,61), seguido pelo de Allen et al. $(1998)(\mathrm{d}=0,88$ e $E F=0,57)$ e Cuenca (1989) $(d=0,87$ e EF $=0,55)$ (Tabela 4). Estes resultados corroboram os de Mendonça et al. (2006), que verificaram melhor desempenho dos métodos de Cuenca (1989) e Allen et al. (1998). O método de Cuenca (1989) também apresentou melhor desempenho no trabalho de Sentelhas \& Folegatti (2003).

Marcussi et al. (2006) encontraram coeficiente de determinação de 0,9928 , entre dados de evapotranspiração calculada pela ECA, utilizando-se o Kp de Allen et al. (1998), e a evapotranspiração calculada com o modelo de Penman-Monteith, em Quillota, no Chile ( $32^{\circ} 53^{\prime} \mathrm{S}, 71^{\circ} 15^{\prime} \mathrm{W}$ e $350 \mathrm{~m}$ de altitude). 
A análise revelou que a adoção de um Kp fixo em 0,7, para a estimativa diária da $\mathrm{ET}_{0}$ com base na ECA, no período anual, apresentou o melhor desempenho. De fato, o Kp médio para o período anual, calculado pela relação entre a $\mathrm{ET}_{0}$ estimada pelo modelo padrão e a obtida a partir da ECA, foi de 0,7021 . Tal resultado confirma que a utilização de um Kp fixo, determinado especificamente para a localidade onde será aplicado, é uma opção prática, eficiente e passível de adoção, especialmente por não haver necessidade de dados meteorológicos e eliminar a etapa de aplicação do método. Sentelhas \& Folegatti (2003) também obtiveram estimativas precisas para período anual, com a utilização de um Kp fixo de 0,71, em Piracicaba (SP).

Ao compararmos o desempenho dos métodos de estimativa do $\mathrm{Kp}$, com base nos valores do coeficiente de correlação (r) proposto por Hopkins (2000) e no índice de desempenho (c) sugerido por Camargo \& Sentelhas (1997) (Tabela 5), verificou-se que a classificação com base no índice "c" se dá dentro de um intervalo de classes menor, sendo capaz de classificar com maior distinção os métodos de estimativa do Kp. Cabe destacar que os valores de " $r$ " e "c" foram equivalentes.

Com base nos coeficiente de correlação (r) e no índice de desempenho (c), os métodos de Pereira et al. (1995), Allen et al. (1998) e Cuenca (1989) foram, respectivamente, os melhores métodos de estimativa

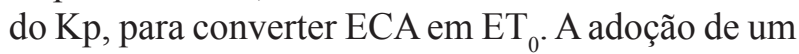
valor fixo para o $\mathrm{Kp}$ apresentou resultados melhores que os obtidos com todos os métodos de estimativa avaliados.

Os métodos que apresentaram o pior desempenho foram o de Snyder (1992) e Doorenbos \& Pruitt (1977). Estes resultados diferem dos obtidos por Conceição (2002), que comparou a $\mathrm{ET}_{0}$ mensal estimada com base na ECA e a de Penman-Monteith, com média de dez anos, e concluiu que o método de Snyder (1992) apresentou o melhor desempenho, com índice de desempenho de 0,883 .

É interessante ressaltar que a maioria das publicações relacionadas ao manejo de culturas irrigadas, com o uso do tanque classe $\mathrm{A}$, recomendam ou trazem apresentado o método baseado na tabela proposta por Doorenbos \& Pruitt (1977). No entanto, neste trabalho, o método apresentou um dos piores desempenhos, quanto à precisão, nas estimativas das $\mathrm{ET}_{0}$ com base na ECA.

Assim como observado no período anual, no período seco, verificou-se desempenho superior dos métodos de Cuenca (1989) e Allen et al. (1998), com valores de $r^{2}$ de 0,679 e 0,662 , respectivamente (Tabela 6). De fato, o modelo proposto por Cuenca (1989) tem se apresentado como o melhor método para estimar o Kp e foi o que apresentou maior coeficiente de determinação, no trabalho de Mendonça et al. (2006), com dados de três anos, no qual comparou $\mathrm{a} \mathrm{ET}_{0}$ diária estimada pela ECA com a do modelo de Penman-Monteith, em baixa altitude, no norte do Estado do Rio de Janeiro. Sentelhas \& Folegatti (2003) também observaram um dos maiores coeficientes de determinação com o modelo de Cuenca (1989), nas condições já mencionadas neste trabalho.

Observou-se melhor desempenho do método de Cuenca (1989) no período seco, o qual apresentou o maior índice de exatidão de Willmott $(0,91)$, sendo este índice relacionado ao afastamento dos valores observados, em relação aos estimados pelo modelo padrão, com valores variando de zero, para nenhuma concordância, a um, para concordância perfeita. Com o método de Cuenca (1989), também foi obtido o menor erro médio absoluto $\left(0,52 \mathrm{~mm} \mathrm{dia}^{-1}\right)$ e o segundo menor erro máximo absoluto $\left(1,71 \mathrm{~mm} \mathrm{dia}^{-1}\right)$, destacando-o como o método mais eficiente de cálculo do Kp. O menor

Tabela 5. Coeficiente de correlação (r), índice de desempenho (c) e classificação do desempenho dos métodos de determinação do $\mathrm{Kp}$, para estimativa diária da $\mathrm{ET}_{0}$ pelo método do tanque classe A, no período anual, em Santo Antônio de Goiás, GO, conforme proposto por Hopkins (2000) e Camargo \& Sentelhas (1997).

\begin{tabular}{lcccc}
\hline \multicolumn{1}{c}{ Método } & $\mathrm{r}$ & Classificação* & $\mathrm{c}$ & Classificação** \\
\hline Doorenbos \& Pruitt (1977) & 0,74 & Muito alta & 0,62 & Mediano \\
Snyder (1992) & 0,72 & Muito alta & 0,59 & Sofrível \\
Cuenca (1989) & 0,79 & Muito alta & 0,68 & Bom \\
Pereira et al. (1995) & 0,83 & Muito alta & 0,72 & Bom \\
Allen et al. (1998) & 0,79 & Muito alta & 0,69 & Bom \\
Kp fixo (0,7) & 0,85 & Muito alta & 0,77 & Muito bom \\
\hline
\end{tabular}

*Hopkins (2000); **Camargo \& Sentelhas (1997). 
Tabela 6. Análise estatística para avaliação dos métodos de estimativa e cálculo do coeficiente do tanque (Kp), para estimar a evapotranspiração de referência diária $\left(\mathrm{ET}_{0}\right)$ pelo método do tanque classe $\mathrm{A}$, no período seco (Santo Antônio de Goiás, GO, 2007).

\begin{tabular}{|c|c|c|c|c|c|c|}
\hline \multirow{2}{*}{ Método } & \multirow{2}{*}{$\mathrm{b}$} & \multirow{2}{*}{$r^{2}$} & \multirow{2}{*}{ d } & EMA & EMAX & \multirow{2}{*}{$\mathrm{EF}$} \\
\hline & & & & 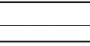 & & \\
\hline Doorenbos \& Pruitt (1977) & 0,93 & 0,62 & 0,87 & 0,58 & 1,78 & 0,52 \\
\hline Snyder (1992) & 0,95 & 0,60 & 0,87 & 0,57 & 1,96 & 0,48 \\
\hline Cuenca (1989) & 0,94 & 0,68 & 0,91 & 0,52 & 1,71 & 0,66 \\
\hline Pereira et al. (1995) & 0,88 & 0,61 & 0,86 & 0,68 & 2,80 & 0,61 \\
\hline Allen et al. (1998) & 0,95 & 0,66 & 0,90 & 0,53 & 1,63 & 0,64 \\
\hline Kp fixo $(0,7)$ & 0,92 & 0,65 & 0,91 & 0,53 & 2,45 & 0,73 \\
\hline
\end{tabular}

b: coeficiente angular; $\mathrm{r}^{2}$ : coeficiente de determinação; d: índice de exatidão de Willmott; EMA: erro médio absoluto; EMAX: erro máximo absoluto; EF: eficiência do método.

EMAX foi de $1,63 \mathrm{~mm}$, obtido com o modelo de Allen et al. (1998) (Tabela 6).

O Kp fixo apresentou a maior eficiência $(0,73)$, sendo superior ao valor obtido com todos os métodos, enquanto o índice de exatidão foi o mesmo do modelo de Cuenca (1989). No entanto, apresentou elevado erro máximo absoluto $(2,45)$, provavelmente pelo fato de a fixação não permitir variações no $\mathrm{Kp}$, em função das condições climáticas vigentes na situação em que se fez a leitura da ECA, o que aumenta a amplitude entre valores extremos de $\mathrm{ET}_{0}$. Adotar um $\mathrm{Kp}$ fixo em 0,7, para estimar a $\mathrm{ET}_{0}$ utilizando tanque classe A, no período seco, nas condições climáticas de Santo Antônio de Goiás (GO), apresentou-se como uma alternativa prática e recomendável, para fins de manejo de irrigação.

Diferentemente do observado no período anual, na seca, o método proposto por Pereira et al. (1995) apresentou o menor índice de exatidão, além de redução na eficiência. Nos dois períodos em questão, o método apresentou o maior erro máximo absoluto. Tal comportamento já era esperado, visto que o método traz, inserido na base de cálculos, a utilização de um valor máximo de Kp pré-fixado em 0,85 , o que propicia a obtenção de coeficientes elevados.
Os coeficientes de correlação obtidos variaram entre 0,78 e 0,85 , respectivamente com os modelos de Snyder (1992) e Pereira et al. (1995). No entanto, de acordo com a classificação proposta por Hopkins (2000), todos os métodos foram classificados com desempenho muito alto (Tabela 7).

Na classificação com base no índice de desempenho proposto por Camargo \& Sentelhas (1997), o modelo de Cuenca (1989) apresentou maior valor para o índice "c" $(0,76)$, sendo o único método classificado com desempenho muito bom. Com o Kp fixado em 0,7, foi obtido o maior coeficiente de correlação $(0,88)$ e o maior índice de desempenho $(0,80)$, classificado como muito bom, o que confirma a possibilidade de adoção do Kp fixo (Tabela 7).

Para o período chuvoso, observou-se que, independentemente do método utilizado no cálculo do $\mathrm{Kp}$, as relações apresentaram baixos valores de coeficientes de determinação (Tabela 8). O mau desempenho provavelmente se deva ao fato de o tanque ter sua superfície evaporante exposta a precipitações. Santos et al. (2008) ressaltaram que uma das limitações no uso de tanques evaporimétricos é a ocorrência de precipitações. De fato, Jensen et al. (1990) observaram que, durante períodos chuvosos,

Tabela 7. Coeficiente de correlação (r), índice de desempenho (c) e classificação do desempenho dos métodos de determinação do $\mathrm{Kp}$, para estimativa diária da $\mathrm{ET}_{0}$ pelo método do tanque classe A, no período seco, em Santo Antônio de Goiás (GO), conforme proposto por Hopkins (2000) e Camargo \& Sentelhas (1997).

\begin{tabular}{lcccc}
\hline \multicolumn{1}{c}{ Método } & $\mathrm{r}$ & Classificação* & $\mathrm{c}$ & Classificação** \\
\hline Doorenbos \& Pruitt (1977) & 0,80 & Muito alta & 0,69 & Bom \\
Snyder (1992) & 0,78 & Muito alta & 0,68 & Bom \\
Cuenca (1989) & 0,84 & Muito alta & 0,76 & Muito bom \\
Pereira et al. (1995) & 0,85 & Muito alta & 0,73 & Bom \\
Allen et al. (1998) & 0,83 & Muito alta & 0,74 & Bom \\
Kp fixo (0,7) & 0,88 & Muito alta & 0,80 & Muito bom \\
\hline
\end{tabular}

*Hopkins (2000); **Camargo \& Sentelhas (1997). 
Tabela 8. Análise estatística para avaliação dos métodos de estimativa e cálculo do coeficiente do tanque (Kp), para estimativa da evapotranspiração de referência diária $\left(\mathrm{ET}_{0}\right)$ pelo método do tanque classe $\mathrm{A}$, no período chuvoso (Santo Antônio de Goiás, GO, 2007).

\begin{tabular}{|c|c|c|c|c|c|c|}
\hline \multirow{2}{*}{ Método } & \multirow{2}{*}{$\mathrm{b}$} & \multirow{2}{*}{$r^{2}$} & \multirow{2}{*}{ d } & EMA & EMAX & \multirow{2}{*}{$\mathrm{EF}$} \\
\hline & & & & 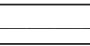 & & \\
\hline Doorenbos \& Pruitt (1977) & 0,82 & 0,30 & 0,78 & 0,85 & 3,01 & 0,35 \\
\hline Snyder (1992) & 0,83 & 0,21 & 0,75 & 0,88 & 3,07 & 0,26 \\
\hline Cuenca (1989) & 0,85 & 0,37 & 0,82 & 0,77 & 2,57 & 0,45 \\
\hline Pereira et al. (1995) & 0,85 & 0,33 & 0,84 & 0,75 & 3,31 & 0,56 \\
\hline Allen et al. (1998) & 0,87 & 0,37 & 0,84 & 0,74 & 2,48 & 0,50 \\
\hline $\operatorname{Kp}$ fixo $(0,7)$ & 0,92 & 0,34 & 0,88 & 0,67 & 2,61 & 0,66 \\
\hline
\end{tabular}

b: coeficiente angular; $\mathrm{r}^{2}$ : coeficiente de determinação; d: índice de exatidão de Willmott; EMA: erro médio absoluto; EMAX: erro máximo absoluto; EF: eficiência do método.

este método apresenta inconsistência na escala diária, principalmente pela dificuldade de separar eventos pluviais da evaporação.

Mesmo com todos os métodos tendo apresentado baixos coeficientes de determinação, os modelos de Allen et al. (1998) e Cuenca (1989) foram os que propiciaram a obtenção dos maiores coeficientes de determinação, no período chuvoso. O valor fixo em 0,7 apresentou baixo desempenho, com $r^{2}=0,34$. Cabe salientar que o Kp médio calculado para o período chuvoso foi de 0,7229 . Assim como observado nos períodos anual e seco, o método de Snyder (1992) apresentou o menor valor de $\mathrm{r}^{2}$.

Os coeficientes angulares obtidos das relações foram menores que aqueles observados nos períodos anual e seco (Tabela 8). Tais valores indicam que, no período chuvoso, o tanque classe A superou, de forma mais acentuada, a $\mathrm{ET}_{0}$ do modelo de Penman-Monteith. O resultado confirma a dificuldade de obtenção de leituras precisas no tanque classe A, em períodos chuvosos, com os valores mais elevados provavelmente devidos a erros provocados pela ocorrência de precipitações, as quais contribuem para leituras com valores elevados de evaporação.
Quanto aos métodos, os valores de Kp tabelados propostos por Doorenbos \& Pruitt (1977) propiciaram a obtenção do menor coeficiente angular, sendo possível inferir que, com o método, foram obtidos valores muito elevados de Kp. O modelo de Snyder (1992) também proveu Kp elevado. Tal resultado explica o fato de este modelo ter apresentado desempenho elevado, em condições de clima semiárido (Gundekar et al. 2008).

Os valores dos índices de exatidão de Willmott(d) foram inferiores aos observados nos períodos anual e seco. Tal resultado evidencia que, no período chuvoso, foram observados valores mais diferenciados de evapotranspiração do tanque, em relação ao modelo padrão, pois este índice está relacionado ao afastamento dos valores observados, em relação aos estimados.

Mesmo com valores dentro de um intervalo amplo $(0,53$ a 0,70$)$, a classificação, quanto ao coeficiente de correlação, não evidenciou diferenças no desempenho dos métodos. Com base nos valores dos índices de desempenho, verificou-se que o método de Pereira et al. (1995) apresentou desempenho superior aos demais métodos, sendo classificado como bom. Os métodos de Cuenca (1989) e Allen et al. (1998) apresentaram desempenho mediano (Tabela 9).

Tabela 9. Coeficiente de correlação (r), índice de desempenho (c) e classificação do desempenho dos métodos de determinação do $\mathrm{Kp}$, para estimativa diária da $\mathrm{ET}_{0}$ pelo método do tanque classe $\mathrm{A}$, no período chuvoso, conforme proposto por Hopkins (2000) e Camargo \& Sentelhas (1997).

\begin{tabular}{lcccc}
\hline \multicolumn{1}{c}{ Método } & $\mathrm{r}$ & Classificação* & $\mathrm{c}$ & Classificação** $^{*}$ \\
\hline Doorenbos \& Pruitt (1977) & 0,71 & Muito alta & 0,56 & Sofrível \\
Snyder (1992) & 0,70 & Muito alta & 0,53 & Sofrível \\
Cuenca (1989) & 0,74 & Muito alta & 0,61 & Mediano \\
Pereira et al. (1995) & 0,79 & Muito alta & 0,66 & Bom \\
Allen et al. (1998) & 0,75 & Muito alta & 0,63 & Mediano \\
Kp fixo (0,7) & 0,79 & Muito alta & 0,70 & Bom \\
\hline
\end{tabular}

*Hopkins (2000); **Camargo \& Sentelhas (1997). 
O Kp fixo apresentou desempenho superior a todos os métodos, com valor do índice de desempenho (c) de 0,70 , sendo classificado com bom desempenho. No entanto, resultou em índice "c" menor que os observados nos períodos anual e seco, respectivamente de 0,77 e 0,80 .

\section{CONCLUSÕES}

1. Para o período anual, o melhor método de estimativa do Kp foi o proposto por Pereira et al. (1995), para as condições climáticas de Santo Antônio de Goiás (GO).

2. Durante o período seco, a melhor estimativa de $\mathrm{ET}_{0}$ com base na ECA foi observada quando utilizados valores de Kp obtidos com o método de Cuenca (1989), para as condições climáticas de Santo Antônio de Goiás (GO).

3. No período chuvoso, os desempenhos dos métodos de cálculo do Kp ficaram comprometidos, em virtude dos erros no manuseio do tanque.

4. A adoção de um Kp fixo e constante de 0,70 resultou em bom desempenho, sendo uma opção bastante prática, porém, faz-se necessária a sua determinação no local onde será aplicado.

\section{REFERÊNCIAS}

ALLEN, R. G. et al. Crop evapotranspiration: guidelines for computing crop water requirements. Rome: FAO, 1998. (Irrigation and drainage paper, 56).

CAMARGO, A. P.; SENTElhas, P. C. Avaliação do desempenho de diferentes métodos de estimativa da evapotranspiração potencial no Estado de São Paulo, Brasil. Revista Brasileira de Agrometeorologia, Santa Maria, v. 5, n. 1, p. 89-97, 1997.

CONCEIÇÃO, M. A. F. Reference evapotranspiration based on class A pan evaporation. Scientia Agricola, Piracicaba, v. 59, n. 3, p. 417-420, 2002.

CUENCA, R. H. Irrigation system design: an engineering approach. New Jersey: Prentice-Hall, 1989.

DOORENBOS, J.; PRUITT, W. O. Crop water requirements. Rome: FAO, 1977. (Irrigation and drainage paper, 24).

FREVERT, D. R.; HILL, R. W.; BRAATEN, B. C. Estimation of FAO evapotranspiration coefficients. Journal of Irrigation and Drainage Engineering, Newark, v. 109, n. 2, p. 265-270, 1983.
GUNDEKAR , H. G. et al. Evaluation of pan coefficient for reference crop evapotranspiration for semi-arid region. Irrigation Science, New York, v. 26, n. 2, p. 169-175, 2008.

HOPKINS, W. G. Correlation coefficient: a new view of statistics. 2000. Disponível em: <http://www.sportsci. org/resource/stats/correl.html>. Acesso em: 12 jul. 2007.

JENSEN, M. E.; BURMAN, R. D.; ALLEN, R. G. Evapotranspiration and irrigation water requirements. New York: ASCE, 1990. (Manuals and reports on engineering practice, 70 ).

MARCUSSI, F. F. N.; SALGADO, E.; WENDLAND, E. Balanço hídrico climatológico comparativo para a cidade de Quillota (Chile) com estimativas de evapotranspiração obtidas pelos métodos de Penman-Monteith e pelo tanque classe A. Irriga, Botucatu, v. 11, n. 43, p. 469-476, 2006.

MENDONÇA, J. C. et al. Coeficientes do tanque classe A para a estimativa da evapotranspiração de referência em Campos dos Goytacazes, RJ. Revista Brasileira de Agrometeorologia, Santa Maria, v. 14, n. 1, p. 123-128, 2006.

PEREIRA, A. R. et al. A model for the class A pan coefficient. Agricultural and Forest Meteorology, Amsterdam, v. 76, n. 1, p. 75-82, 1995.

PEREIRA, A. R.; VILLA NOVA, N. A.; SEDIYAMA, G. C. Evapotranspiração. Piracicaba: Fealq, 1997.

SANTOS, F. X. et al. Desempenho de lisímetro de pesagem hidráulica de baixo custo no semiárido nordestino. Engenharia Agrícola, Jaboticabal, v. 28, n. 1, p. 115-124, 2008.

SENTELHAS, P. C. Agrometeorologia aplicada à irrigação. In: MIRANDA, J. H.; PIRES, R. C. M. Irrigação. Piracicaba: Funep, 2001. p. 63-120.

SENTElhas, P. C.; FOLEGATTI, M. V. Class A pan coefficients $(\mathrm{Kp})$ to estimate daily reference evapotranspiration (ETo). Revista Brasileira de Engenharia Agrícola e Ambiental, Campina Grande, v. 7, n. 1, p. 111115, 2003.

SNYDER, R. L. Equation for evaporation pan to evapotranspiration conversion. Journal of Irrigation and Drainage Engineering of ASCE, New York, v. 118, n. 6, p. 977-980, 1992.

WILLMOTT, C. J. et al. Statistics for the evaluation and comparison of models. Journal of Geophysical Research, Ottawa, v. 90, n. C5, p. 8995-9005, 1985.

ZACHARIAS, S.; HEATWOLE, C. D.; COAKLEY, C. W. Robust quantitative techniques for validating pesticide transport models. Transactions of the ASAE, St. Joseph, v. 39, n. 1, p. 47-54, 1996. 